\title{
Solving boundary layer problem using fifth order block method
}

\begin{abstract}
A fifth order block method of Adamôs type is presented to obtain the numerical solution of the boundary layer problem. The boundary layer problem we handle in this research is nanofluid over a moving surface in a flowing fluid. It is modelled as a system of combination of third order and second order differential equations subject to the two point boundary conditions. The block method will implemented with variable step size strategy and multiple shooting technique to solve this boundary layer problem. Two approximate solutions will obtain simultaneously using the same back value. The boundary layer problem will be solved directly without reducing to the system of first order equations. The numerical results are presented and compared to the existing method.
\end{abstract}

Keyword: Boundary layer problem; Fifth-order block method; Multiple shooting techniques 